\title{
Scattering Regimes for Underwater Optical Wireless Communications using Monte Carlo Simulation
}

\author{
F. Jasman' ${ }^{1}$ A.M. Zaiton ${ }^{2}$, Z. Ahmad ${ }^{3}$, Z. Rihawi ${ }^{4}$ \\ ${ }^{1}$ Faculty of Engineering and Computing, First City University College, Selangor, Malaysia \\ ${ }^{2}$ Faculty of Electronic \& Computer Engineering, Universiti Teknikal Malaysia Melaka, Malaysia \\ ${ }^{3}$ Faculty of Engineering, Environment and Computing, Coventry University, UK \\ ${ }^{4}$ Warwick Manufacturing Group (WMG), University of Warwick, UK
}

\begin{tabular}{l} 
Article Info \\
\hline Article history: \\
Received Jan 30, 2018 \\
Revised Apr 2, 2018 \\
Accepted Apr 9, 2018
\end{tabular}

\section{Keyword:}

Channel bandwidth

Monte Carlo

Scattering regimes

Transition point

Underwater optical wireless

\begin{abstract}
Optical wireless communications has shown tremendous potential for underwater applications as it can provide higher bandwidth and better security compared to acoustic technologies. In this paper, an investigation on scattering regimes for underwater links using Monte Carlo simulation has been presented.While the focus of this paper is on diffuse links, the simulation results of collimated links is also provided for comparison purpose. Three types of water namely clear, coastal and turbid water are being used in the simulation. It is shown that the effect of scattering on the path loss cannot be accurately modeled by the existing channel model; ie. Beers-Lambert (BL) law. It has been shown that the distance at which the unscattered light drops to zero can be used to estimate the transition point for the scattering regimes in case of diffuse links. The transition point for diffuse links in coastal water and turbid water can be estimated to be around $22 \mathrm{~m}$ and $4 \mathrm{~m}$ respectively. Further analysis on the scattering order probability at different scattering regimes illustrates how scattering is affected by beam size, water turbidity and distance. From the frequency response plot, it is estimated that the bandwidth of several order of $\mathrm{GHz}$ can be achieved when the links are operating in the minimal scattering region and will reduce to several hundreds of $\mathrm{MHz}$ when the link is operating in multiple scattering region.
\end{abstract}

Copyright $\odot 2018$ Institute of Advanced Engineering and Science. All rights reserved.

\section{Corresponding Author:}

Faezah Jasman,

Faculty of Engineering and Computing,

First City University College,

Persiaran Bandar Utama, 47800 petaling Jaya, Selangor, Malaysia.

Email: faezah.jasman@firstcity.edu.my

\section{INTRODUCTION}

Optical wireless communications (OWC) which offer high bandwidth ( GHz), but only over short ranges $(<200 \mathrm{~m})$ is seen as a complementary technology to the acoustic technology for underwater applications. Historically, research on using lasers for undersea communications has started as early as the 1970s [1]. Specifically, wavelengths in the blue/green region have been used as they exhibit the minimum attenuation underwater. Recently, data rate of up to $5.5 \mathrm{Gbps}$ has been demonstrated over the range of $26 \mathrm{~m}$ [2]. Despite the high data rate achieved by a laser based links, they face a considerable challenge to maintain accurate pointing and tracking since laser beams are highly collimated. Thus, several investigations to develop a diffuse system based on LED to ease the strict pointing requirements have been conducted. An experiment using an omnidirectional transmitter at $40 \mathrm{Mbps}$ over $10 \mathrm{~m}$ has been reported in [3].

One of the main challenges to implement an underwater OWC (UOWC) is the effect of scattering that causes temporal dispersions which eventually limit the bandwidth of the links. Many works have been 
done to understand and characterise the effect of scattering. In [4], a categorisation of the energy transport region for the blue-green pulsed laser through fog in the atmosphere has been proposed. In [5], [6], an equation is used to estimate the transition point from an unscattered region to a multiple scattering regions, which is beneficial to predict the distance where the bandwidth will be limited. It is predicted that the channel bandwidth of several order of $\mathrm{GHz}$ can be supported when the link is operating in minimal scattering region where unscattered light dominates. An experimental work conducted in [6] has shown that the transition point obtained for collimated beam matched the work in [4]. Several papers also highlighted the importance of identifying the operating region of underwater links [7], [8]. For example, in the design of relay assisted communication or multi-hop cooperative communication, it is crucial to determine the placement of the relay nodes in order to optimize the overall end-to-end links. Thus, this paper will focus on the simulation of diffuse links to investigate the scattering behavior in three types of water as none of the previous work investigates the scattering regime for the diffuse links. The remainder of this paper is organised as follows: Subsection 1.1 and 1.2 present a brief introduction to underwater optical properties and channel model. Section 2 describes the proposed system based on Monte Carlo (MC) simulation. Afterwards, numerical results and key findings are discussed in Section 4. Finally, Section 5 concludes the work.

\subsection{Optical properties}

The two major optical properties that attenuate water are absorption and scattering. Absorption is a process when the photon energy is lost due to the transfer of energy during the interaction with water molecules and particles. Scattering is a process where a photon's path is deflected due to the interaction with a particulate matter in water [9].This process causes spatial dispersion that reduces the signal-to-noise ratio (SNR) due to the light from the source spreads out in space and causes a reduction of the number of photons collected at the receiver. Additionally, scattering also causes temporal dispersion which results from different time of arrival of photons at the receiver. This will limit the channel bandwidth available for communication due to the introduction of inter-symbol-interference [10]. Both of these effects are wavelength dependent and are generally represented by the attenuation coefficient $c$ which is the summation of absorption coefficient $a$, and scattering coefficient $b$. Coefficient values for different water have been established in the literature and are shown in Table 1 [11], [12].

Table 1. Optical Properties of three Types of Water

\begin{tabular}{cccc}
\hline Water type & $a\left(\mathrm{~m}^{-1}\right)$ & $b\left(\mathrm{~m}^{-1}\right)$ & $c\left(\mathrm{~m}^{-1}\right)$ \\
\hline Clean water & 0.114 & 0.037 & 0.151 \\
Coastal water & 0.179 & 0.219 & 0.398 \\
Turbid water & 0.366 & 1.824 & 2.19 \\
\hline
\end{tabular}

\subsection{Channel model}

One of the challenges in underwater environment is to establish a universal analytical model to predict the performance of UOWC links. While the work on this aspect continues, several researchers have utilised Beer-Lambert (BL) law due to its simplicity. By using BL law, the received power can be calculated as [9].

$$
P=P_{o} \exp (-c(\lambda) z)
$$

Where $P_{O}$ is the transmitted power, $z$ is the path length and $c(\lambda)$ is the beam attenuation coefficient. The beam attenuation coefficient, $c(\lambda)$ is calculated using

$$
c(\lambda)=a(\lambda)+b(\lambda)
$$

Where $a(\lambda)$ is the absorption coefficient and $b(\lambda)$ is the scattering coefficient. It can be seen from Equations (1) and (2) that the BL law only considers the attenuation due to the absorption and the scattering and does not consider any re-scattered light that contributed to the received power. As a result, it underestimates the power received in the high scattering environment as some of the re-scattered light will be collected by the receiver. Furthermore, BL law only applies to collimated beams in LOS links. Another alternative in modeling the underwater channel is by using MC numerical methods which is regarded as a powerful tool due to its flexibility and accuracy. Several works using MC simulation in modeling and 
characterization of the UOWC have been reported. An investigation on the path loss and the channel bandwidth performance of collimated links has been reported in [5], [13] and [14]. It is shown that a MC simulation can predict the performance and capacity more accurately. Similarly, several works on modelling diffuse links have been reported. For example in [15] and [16], MC simulation has been utilised to model the impulse response which was used to verify the analytical equation of the impulse response proposed.

\section{RESEARCH METHOD}

\subsection{Simulation setup}

In this paper, MC simulation is used to model the diffuse links where the light beam is modelled as the continuous propagation of a large group of photons. A set of probability rules and random variables are used to model the initial beam distributions, path length and scattering angle. For a detailed description of Monte Carlo simulation, readers can refer to [5] and [17]. Three types of water have been considered in this simulation: clear water, coastal water and turbid water. The distance between the source and the receiver was varied between $1 \mathrm{~m}$ to $50 \mathrm{~m}$. Two sizes of beam divergence for diffuse sources are used namely $15^{\circ}$ and $30^{\circ}$. Other simulation parameters were set as listed in Table 2. To simplify the simulation, the effect of turbulence, background radiation, noises and surface waves has been neglected.

Table 2. Simulation Parameters

\begin{tabular}{cc}
\hline Parameter & Value \\
\hline Wavelength & $514 \mathrm{~nm}$ \\
Beam width & $3 \mathrm{~mm}$ \\
Collimated source beam divergence (full-angle) & $1.5 \mathrm{mrad}$ \\
Diffuse source beam divergence (full-angle) & $15^{\circ}, 30^{\circ}$ \\
Receiver aperture diameter & $10 \mathrm{~cm}$ \\
Receiver field-of-view (FOV) & $180^{\circ}$ \\
\hline
\end{tabular}

\section{RESULTS AND ANALYSIS}

\subsection{Path loss performance}

Figure 1(a) shows the path loss performance for collimated and diffused links in clear water and coastal water as the distance is varied from $5 \mathrm{~m}$ to $50 \mathrm{~m}$. For turbid water, a separate plot has been presented as the maximum distance studied is up to $20 \mathrm{~m}$ only. Firstly, it can be seen that, both in clear water and coastal water, the path loss performance for diffuse links did not match BL law due to the huge power loss caused by the spreading and geometric loss. A totally different behaviour is observed for turbid water, where the path loss performance is better than what is predicted by BL law for a distance greater than $5 \mathrm{~m}$ as shown in Figure 1(b). This is because, in MC simulations, both unscattered and re-scattered light are collected at the receiver which results in larger received power.
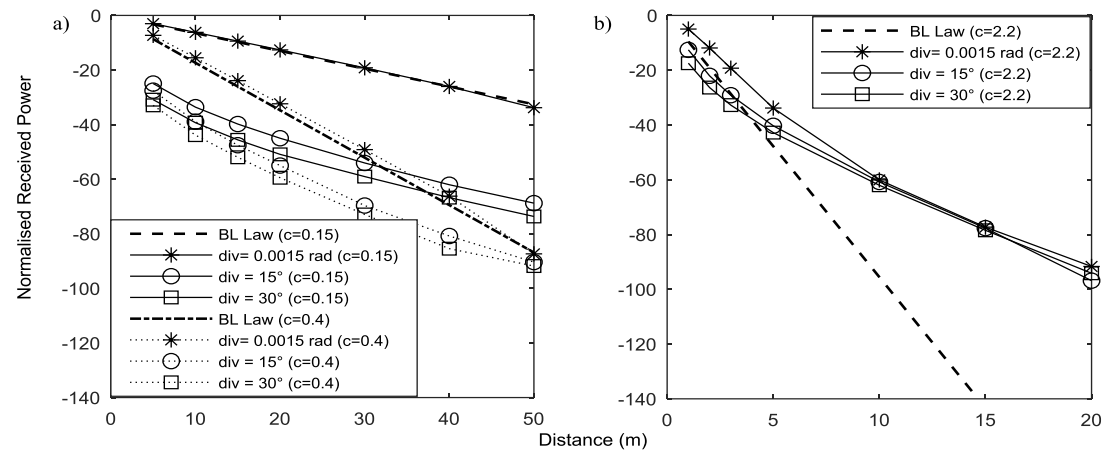

Figure 1. Path loss performance of various beam sizes in (a) clear and coastal water and (b) turbid water

\subsection{Percentage of unscattered light}

Figure 2 shows the percentage of the unscattered light that contributed to the total power reception as the distance is varied. It is apparent that the unscattered light dominates in all types of links in clear water with collimated link showing almost $100 \%$ contributions. However, scattering has shown a significant impact when diffuse links are used in both coastal and turbid water as the percentage of unscattered light is less than 
$50 \%$ even for short distance $(10 \mathrm{~m})$. In contrast, collimated link still shows high contributions of unscattered light in coastal water up to $50 \mathrm{~m}$.

From this plot, the distance at which the unscattered light drops to approximately zero is proposed to be used as the transition point between the minimally scattering and the multiple scattering region. Apparently, in clear water, all three beams show a high percentage of unscattered light up to a distance of $50 \mathrm{~m}$. Thus, the transition point for clear water cannot be determined as the study is limited to $50 \mathrm{~m}$. More simulations can be done at a longer distance to investigate this in the future. Alternately, in coastal and turbid water, it can be seen that the transition point for both the diffuse links are estimated to be $22 \mathrm{~m}$ and $4 \mathrm{~m}$ respectively. This is slightly shorter than the transition point for the collimated link which is found to be $50 \mathrm{~m}$ and $7 \mathrm{~m}$ for coastal water and turbid water respectively.

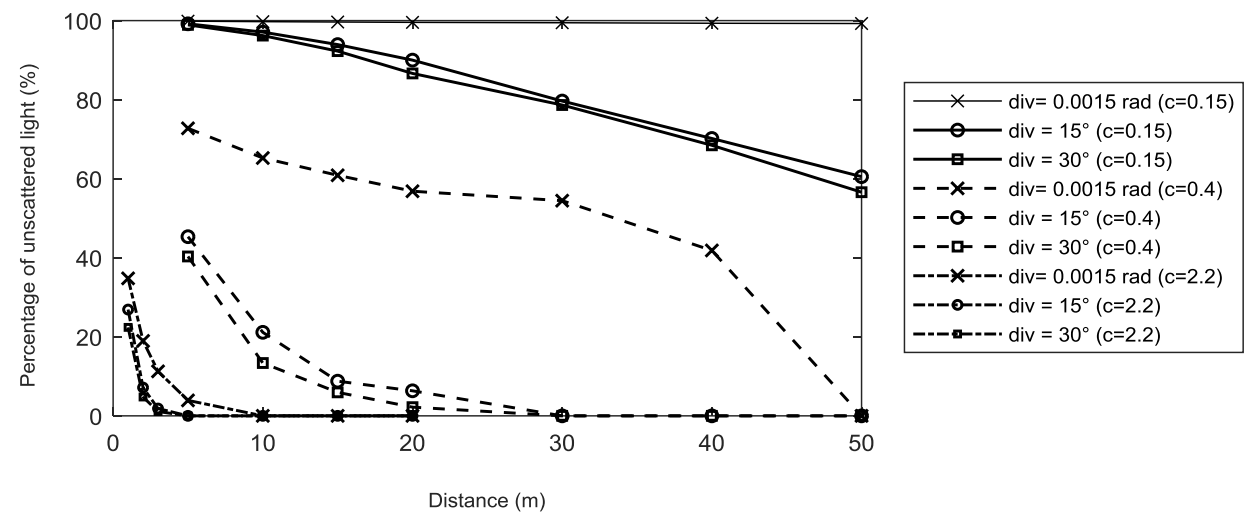

Figure 2. The percentage of the unscattered light that contributes to the total power reception

\subsection{Scattering order probability}

In the previous section, it has been illustrated that the scattering is more significant in coastal and turbid water as shown by the reduction of the percentage of the unscattered light. Thus, it is interesting to investigate the nature of the scattering behaviour of the light that is collected at the receiver by finding the scattering order which is defined as the number of times the received light has been scattered along their propagation path. Unscattered light will have scattering order of 0 indicating that they are not scattered. Three distances are chosen to represent the minimal scattering region, the transition point, and multiple scattering region for both diffuse links as summarized in Table 3. Although we cannot demonstrate the scattering behaviour for the three regions in clear water, we included the plot to show the scattering behaviour of various links as distance is increased as shown in Figure 3.

Figure 4 and Figure 5 illustrate the scattering order histogram for different scattering regimes for coastal water and turbid water respectively. The relation between scattering order with the beam divergence sizes, distance and types of water can be found in the figure. Firstly, it can be seen that the probability of higher scattering order increases at longer distance, particularly if diffuse links are used. This indicates that the scattering is significantly affected by the size of the beam divergence. It is also illustrated that in the minimal scattering region in both coastal and turbid water, a higher order of multiple scattering exists in diffuse links compared to a collimated link. At the transition point, both diffuse links are dominated by lower order scattering $(\mathrm{n}<5)$ while in multiple scattering region, most of the light has been scattered by more than 5 times. From the plot it is also apparent that the turbid water shows more scattering effect even when the links are operating in minimal scattering region.

Table 3. Summary of select distance in Coastal and Turbid Water

\begin{tabular}{cccc}
\hline Water Types & Minimal scattered region & Transition point & Multiple scattered region \\
\hline Coastal water & $15 \mathrm{~m}$ & $22 \mathrm{~m}$ & $50 \mathrm{~m}$ \\
Turbid water & $1 \mathrm{~m}$ & $3 \mathrm{~m}$ & $7 \mathrm{~m}$ \\
\hline
\end{tabular}



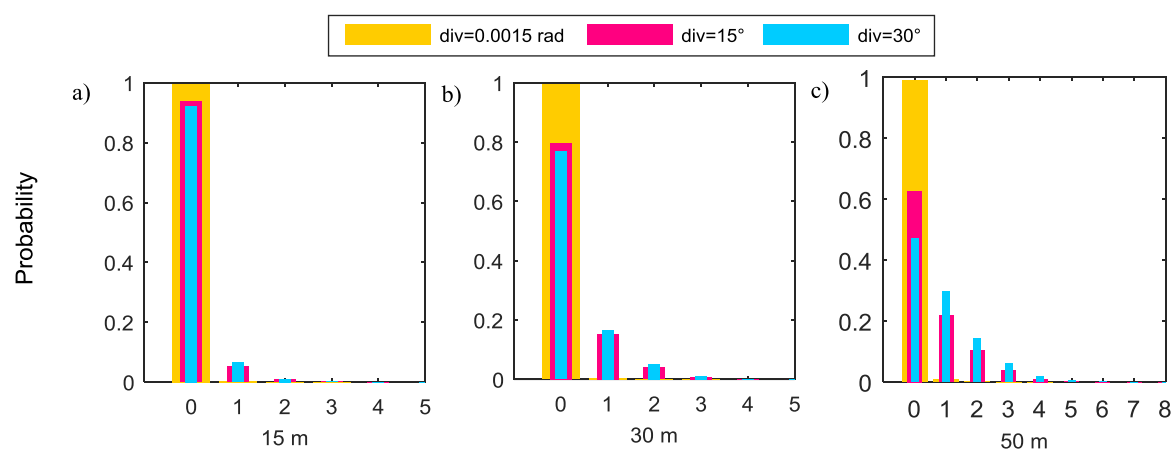

Scattering Order

Figure 3. Scattering order histogram in clear water for various beam sizes at (a) $15 \mathrm{~m}$, (b) $30 \mathrm{~m}$ and (c) $50 \mathrm{~m}$

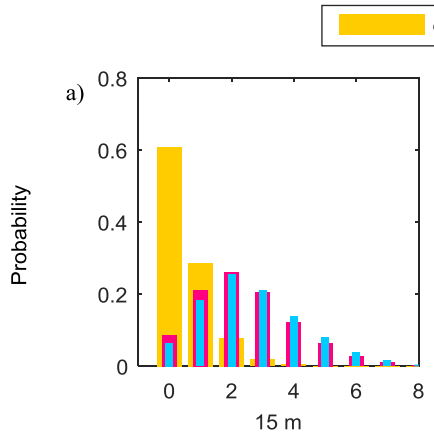

$\operatorname{div}=0.0015 \mathrm{rad}$ $\operatorname{div}=15^{\circ}$ $\operatorname{div}=30^{\circ}$
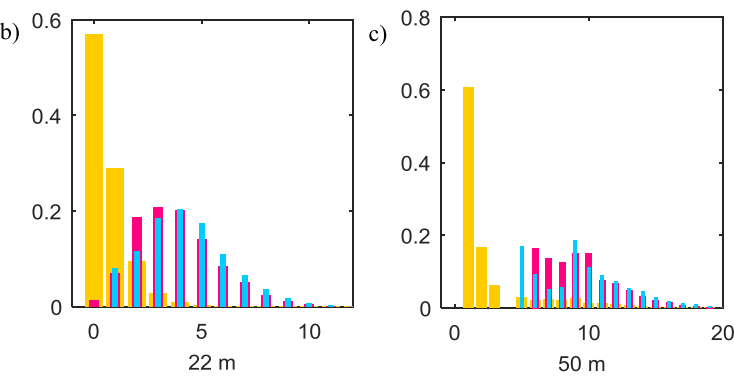

Scattering Order

Figure 4. Scattering order histogram in coastal water for various beam sizes at (a) $15 \mathrm{~m}$, (b) $30 \mathrm{~m}$ and (c) $50 \mathrm{~m}$

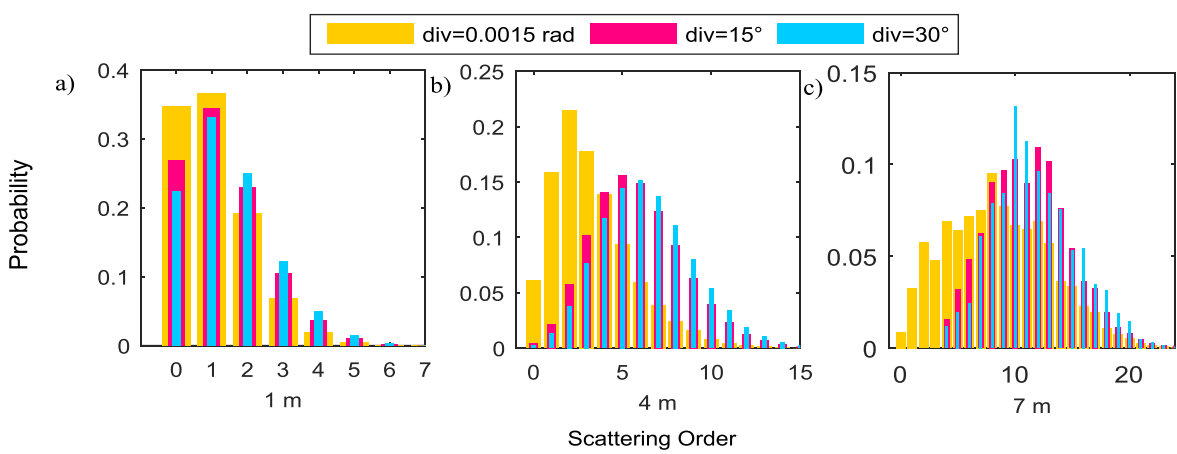

Figure 5. Scattering order histogram in turbid water for various beam sizes at (a) $1 \mathrm{~m}$, (b) $4 \mathrm{~m}$ and (c) $7 \mathrm{~m}$

\subsection{Frequency response}

In order to investigate the temporal dispersion of the links in various scattering regimes, the channel bandwidth has been estimated using a frequency response plot (i.e. drops by $3 \mathrm{~dB}$ ). In this plot, only collimated link and a diffuse link utilizing the full divergence angle of $15^{\circ}$ are shown to simplify the plot. Figure 6(a), Figure 6(b), and Figure 6(c) show the frequency responses of both links in clear, coastal and turbid water respectively. In clear water, the bandwidth supported by the links cannot be calculated due to the fact that the light is dominated by the unscattered light indicating that a bandwidth in the order of $\mathrm{GHz}$ is possible. For both coastal water and turbid water, it is shown that the bandwidth reduces from GHz range to several hundreds of $\mathrm{MHz}$ when the operating regime changes from minimal scattering to multiple scattering regions. It should be noted that at the transition point, several order of $\mathrm{GHz}$ can still be supported if a considerable amount of unscattered and single scattered light are present. 

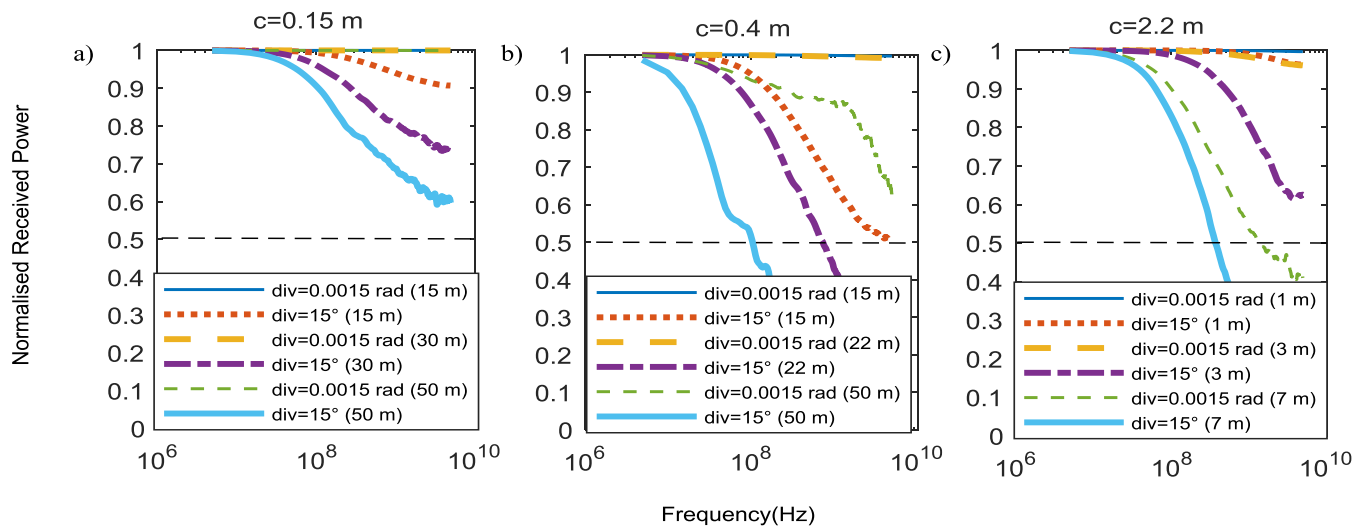

Figure 6. Frequency responses of various beam sizes in (a) clear water, (b) coastal water and (c) turbid water

\section{CONCLUSION}

In this paper, MC based simulation has been utilised to investigate the nature of scattering behaviour of a diffuse link in various types of water. Firstly, a more accurate path loss model can be obtained by using a MC simulation compared to the simplified BL law. This is because MC simulation considers the re-scattered light in the calculation of the received power. The percentage of unscattered light collected at the receiver not only show how scattering plays an important role in transmission link but also provides an important information in the prediction of the transition point between minimal scattering and multiple scattering region. It can be said that a diffuse link can operate in minimal scattering regimes up to distance of $22 \mathrm{~m}$ in coastal water and $4 \mathrm{~m}$ in turbid water. To understand this further, the scattering order behaviour of the links has been evaluated at three regions in order to demonstrate how scattering is affected by beam sizes, distance and water types. Finally, the channel bandwidth that can be supported in different regions has been investigated. In minimal scattering region, bandwidth up to several $\mathrm{GHz}$ can be supported and reduces to several hundred of $\mathrm{MHz}$ in multiple scattering region. It can be seen that by classifying the operating region based on scattering order, a better method in predicting the performance of OWC links has been obtained. It is also believed that the transition point proposed in this paper can be useful in the characterisation of the analytical model reported in [7], where the exact distance at which the model is valid is unknown. Thus, the results presented here will provide some insights into this issue.

\section{REFERENCES}

[1] S. Karp, "Optical Communications Between Underwater and Above Surface (Satellite) Terminals," IEEE Transactions on Communications," vol. 24, no. 1, pp. 66-81, 1976.

[2] Y. Chen, et al., "26 m/5.5 Gbps air-water optical wireless communication based on an OFDM-modulated 520-nm laser diode," Opt. Express, vol. 25, no. 13, pp. 14760-14765, 2017.

[3] G. Baiden, et al., "Paving the way for a future underwater omni-directional wireless optical communication systems," Ocean Engineering, vol. 36, no. 9, pp. 633-640, 2009.

[4] G. C. Mooradian, et al., "Blue-green pulsed propagation through fog," Applied Optics, vol. 18, no. 4, pp. 429-441, 1979.

[5] W. C. Cox Jr, "Simulation, Modeling, and Design of Underwater Optical Communication Systems," PhD Thesis, Dept. Elect. and Comp. Eng, North Carolina State Univ, 2012.

[6] B.Cochenour, et al., "Temporal response of the underwater optical channel for high-bandwidth wireless laser communications," IEEE Journal of Oceanic Engineering, vol. 38, no. 4, pp.730-742, 2013.

[7] S. Tang, et al., "Impulse Response Modeling for Underwater Wireless Optical Communication Links," IEEE Transactions on Communications, vol. 62, no. 1, pp. 226-234, 2014.

[8] Z. Zeng, et al., "A survey of underwater optical wireless communications," IEEE Communications Surveys \& Tutorials, vol. 19, no. 1, pp. 204-238, 2017.

[9] S. Arnon, et al., "Advanced optical wireless communication systems, "Cambridge University Press, 2012, pp. 203.

[10] B. Cochenour and L. Mullen, "Channel response measurements for diffuse non-line-of-sight (NLOS) optical communication links underwater," in OCEANS, 19-22 Sept 2011, pp. 1-5.

[11] C. D. Mobley and C. D. Mobley, "Optical Properties of Water," in Light and water: Radiative transfer in natural waters, vol. 592: Academic Press, San Diego, CA, 1994, pp.109.

[12] T. J. Petzold, "Volume scattering functions for selected ocean waters," Scripps Institute of Oceonography, San Diego, CA, Tech. Rep. Oct 1972. 
[13] J. Li, et al., "Monte Carlo study on pulse response of underwater optical channel," Optical Engineering, vol. 51, no. 6, pp. 066001-1, 2012.

[14] W. Cox and J. Muth, "Simulating channel losses in an underwater optical communication system," Journal of the Optical Society of America A, vol. 31, no. 5, pp. 920-934, 2014.

[15] C. Gabriel, et al., "Monte-Carlo-based channel characterization for underwater optical communication systems," IEEE/OSA Journal of Optical Communications and Networking, vol. 5, no. 1, pp. 1-12, 2013.

[16] Y. Dong, et al. "On impulse response modeling for underwater wireless optical MIMO links," in IEEE/CIC Int. Conf. on Communications (ICCC), Shanghai, China, 13-15 Oct, 2014, pp. 151-155.

[17] R.A. Leathers, et al., "Monte Carlo Radiative Transfer Simulations for Ocean Optics: A Practical Guide," Naval Research Lab., Washington, DC, Tech.Memo. NRL/MR/5660—04-8819, Sept 1, 2004.

\section{BIOGRAPHIES OF AUTHORS}
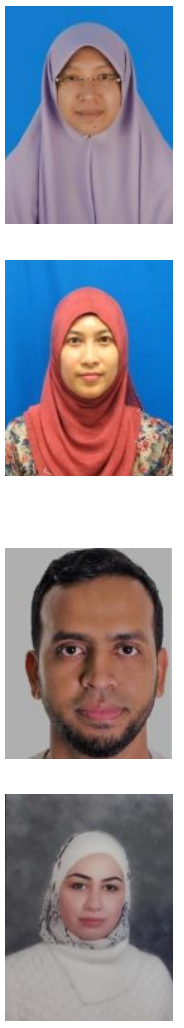

Faezah Jasman received the B.Eng (Hons) degree in electronics engineering from Multimedia University, in 2000, the M.Sc. degree in communication and network engineering from Universiti Putra Malaysia, in 2009, and the Ph.D. degree in communication engineering from University of Warwick, UK, in 2016. She is currently working as a Lecturer at the Faculty of Engineering and Computing, First City University College, Selangor. Her research interests include underwater optical wireless communications as well as visible light communications.

Zaiton Abdul Mutalip received the B.Eng (Hons) degree in electronics and telecommunication engineering from Universiti Malaysia Sarawak, in 2000, the M.Sc. degree in optical communication systems and network from University of Hertfordshire, in 2008, and the Ph.D.degree in communication engineering from University of Warwick, UK, in 2016. At present she is a senior lecturer at the Faculty of Electronic \& Computer Engineering at Universiti Teknikal Malaysia Melaka. Her main research interests are in the field of optical wireless communications, in-vehicle networks as well as photonics.

Zahir Ahmad is a Lecturer of Electrical/Electronic Engineering at Coventry University, UK. He received his $\mathrm{PhD}$ in Optical Wireless Communications at the University of Warwick, where he developed an underwater visible light communication system for sensor applications. Previously, Zahir had participated in the development of ITU-T G.8032 protocols. Zahir has published papers related to the communication field and his current research focus is free space optical wireless communications, $\mathrm{V} 2 \mathrm{~V}$ visible light communication, underwater visible light communications etc.

Zeina Rihawi was born in Syria. She received the B.Sc. degree in Electronic Engineering Communication Department from Electric and Electrical Engineering Faculty, Aleppo University, Aleppo, Syria, in 2007, the M.Sc. degree in Electronic Systems with Communications with distinction from University of Warwick, UK, in 2010, and the Ph.D degree at the School of Engineering, University of Warwick, UK in 2016. She worked as teaching assistant for three semesters (2008-2009) in Communication Department, Electric and Electrical Engineering Faculty, University of Aleppo, Syria. After finishing PhD, she worked as Engineering tutor in Coventry University College for one year. Currently, she is working as Senior Teaching Fellow at Warwick Manufacturing Group, Warwick University. Her main research interests are in the field of optical wireless communications, in-vehicle networks and multimedia communications. 